\title{
Gangrena de Fournier: generalidades
}

\author{
Fournier's gangrene: generalities
}

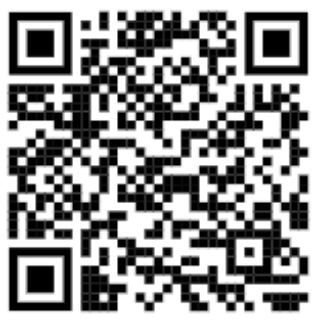

\author{
${ }^{1}$ Dra. Tatiana Vargas Rubio \\ Investigadora independiente, Heredia, Costa Rica \\ (D) https://orcid.org/0000-0002-4635-7975 \\ ${ }^{2}$ Dra. Sofía de los Ángeles Mora Agüero \\ Investigadora independiente, Heredia, Costa Rica \\ (D) https://orcid.org/0000-0001-5032-6760 \\ ${ }^{3}$ Dra. Ana Sofía Zeledón Aguilera \\ Investigadora independiente, Heredia, Costa Rica \\ (D) https://orcid.org/0000-0001-6542-4240 \\ CORREGIDO \\ ACEPTADO \\ $11 / 04 / 2019$ \\ $30 / 04 / 2019$
}

RECIBIDO

$03 / 04 / 2019$

\section{RESUMEN}

La gangrena de Fournier es una patología poco frecuente, más frecuente en los hombres que en las mujeres, pero con índices de mortalidad bastante elevados. La cual se caracteriza por una fascitis necrotizante rápidamente progresiva del área perianal y genitourinaria, asociada a diversos factores de riesgo. Dada la elevada mortalidad y complicaciones es fundamental conocer los hallazgos clínicos y de imagen que puedan sugerir esta patología para un diagnóstico oportuno, siendo fundamental el tratamiento temprano y agresivo de esta patología.

${ }^{1}$ Médico general, graduada de la

PALABRAS CLAVE: gangrena de Fournier; infecciones de los Universidad de Ciencias tejidos blandos; perineo. Médicas (UCIMED). tavaru0309@gmail.com

\section{ABSTRACT}

Fournier's gangrene is a rare pathology, more frequent in men than in ${ }^{2}$ Médico general, graduada de la Universidad de Ciencias Médicas (UCIMED), código médico 14657 sofim02291@gmail.com women, but with quite high mortality rates. Which is characterized by rapidly progressive necrotizing fasciitis of the perianal and genitourinary area, associated with various risk factors. Given the high mortality and complications, it is essential to know the clinical and imaging findings that may suggest this pathology for an

${ }^{3}$ Médico general, graduada de la Universidad de Ciencias Médicas (UCIMED), código médico 14569 opportune diagnosis, for the early and aggressive treatment of this pathology.

sofiazeledon@gmail.com

KEYWORDS: Fournier gangrene; soft tissue infections; perineum. 


\section{INTRODUCCIÓN}

La gangrena de Fournier (GF), es una fascitis necrotizante rápidamente progresiva del área perianal y genitourinaria que afecta más a hombres que a mujeres. Las tasas de mortalidad reportadas por esta causa son altas, por lo que es importante conocer los hallazgos clínicos que sugieran esta patología para su adecuado diagnóstico y tratamiento (1).

La GF tiene un fondo histórico que data desde la era hipocrática. Desde 1871, Joseph Jones hizo las primeras descripciones de esta enfermedad. Posteriormente en 1883, Jean Alfred Fournier, médico dermatólogo francés, especialista en enfermedades de transmisión sexual, reportó un tipo de gangrena fulminante como consecuencia de su experiencia con cinco pacientes masculinos con lesiones en sus genitales; es tras el descubrimiento de este médico que se llamó "gangrena de Fournier" a esta patología. Posteriormente a principios del siglo XIX, fueron llamadas "úlceras malignas" o "gangrena pútrida". Luego en 1952, B. Wilson lo llamó "Fascitis Necrotizante" basándose en una descripción detallada de la infección y la necrosis de la fascia (2).

Esta revisión bibliográfica tiene como objetivo exponer los aspectos generales sobre la gangrena de Fournier, su etiología, características clínicas, métodos diagnósticos, clasificación de severidad y su tratamiento, ya que a pesar de ser un padecimiento raro en la población en general, tiene altas tasas de mortalidad en los pacientes que la presentan.

\section{MATERIALES Y MÉTODOS}

Para esta revisión bibliográfica se buscó información en diversas fuentes, entre ellas Pubmed y Scientific Electronic Library Online, bajo los términos "fascitis necrotizante" y "gangrena de fournier". La búsqueda fue enfocada en las generalidades del tema, así como los puntos importantes en cuanto a diagnóstico y manejo. Se tomaron en cuenta artículos en español e inglés, además de artículos actualizados en los últimos años, una vez clasificada la información se redactó esta revisión.

\section{EPIDEMIOLOGÍA}

Es una enfermedad rara, especialmente en mujeres, ya que la incidencia reportada es $1.6 / 100,000$ de la población masculina; los casos masculinos superan en número a los casos femeninos en una proporción de 10:1. La GF se observa principalmente en hombres de 40 a 50 años (1).

El mayor número de casos publicados ha sido realizado por Eke entre 1950 y 1999 utilizando la base de datos de Medline, en los que se evidenció un registro promedio de 97 casos por año, para un total de 1.726 casos. Pese al progreso en el cuidado clínico de pacientes con GF, la literatura reporta una alta tasa de mortalidad, alcanzando incluso el 20$43 \%$, sin embargo esta es variable (4).

\section{FACTORES DE RIESGO}

Existen varias condiciones relacionadas con la aparición de la enfermedad. La diabetes mellitus ha sido identificada como la comorbilidad más prevalente en los pacientes con GF, ya que la 
hiperglucemia afecta de manera directa las funciones de quimiotaxis, fagocitosis y respuesta inmune mediada por células (4).

Sin embargo, también se han identificado otros factores predisponentes como: obesidad, déficit neurológico, alcoholismo crónico, neoplasias malignas, consumo crónico de corticoesteroides, desnutrición, infección por el VIH, enfermedad vascular periférica e hipertensión arterial esencial (4).

\section{ETIOLOGÍA}

El informe inicial de esta patología suponía que la etiología de GF era idiopático; sin embargo ahora se sabe que la GF es el resultado de una infección polimicrobiana (Tipo I), que incluye patógenos tanto aerobios como anaerobios (3).

La fascitis necrotizante en términos globales se puede dividir en dos categorías microbiológicas: infección polimicrobiana (tipo I, la cual incluye la Gangrena de Fournier) y la monomicrobiana (tipo II) (5).

La infección necrotizante polimicrobiana (tipo I) es causada por bacterias aerobias y anaerobias, por lo general al menos una especie anaerobia como por ejemplo Bacteroides, Clostridium o Peptostreptococcus; estos se aíslan en combinación con algúna enterobacteria como Escherichia coli, Enterobacter, Klebsiella o Proteus y uno o más estreptococos anaerobios facultativos, que no sean del grupo $A$, como por ejemplo el S. agalactiae (6).

Concluyendo así que la GF generalmente es causada por organismos facultativos tales como E. coli, Klebsiella, Enterococos; junto con anaerobios tales como Bacteroides, Fusobacterium, Clostridium, anaerobios o Estreptococos Microaerófilos (6).

\section{FISIOPATOLOGÍA}

La GF se caracteriza porque suele haber un punto de entrada en la piel que genera un desequilibrio entre inmunidad del huésped y la virulencia de los microorganismos, produciendo así enzimas como la colagenasa, la lecitinasa y exotoxinas, que llevan a una rápida multiplicación de microorganismos, a la destrucción de las barreras con la consiguiente dispersión de la enfermedad (7).

Las bacterias invasoras causan trombosis de los vasos que se encuentran en la hipodermis, lo que lleva a isquemia tisular la cual, además, es agravada por la presencia de edema $(7,8)$. La isquemia tisular promueve la diseminación infecciosa que posteriormente conduce a necrosis de la piel. Esto también explica los fenómenos de dolor intenso que se observan, especialmente cuando las ramas de los nervios también se ven afectadas. Tales casos también pueden mostrar signos de hipoestesia / anestesia regional. La linfangitis y la linfadenopatía son raras debido a la trombosis de los vasos. Por otra parte el gas formado por bacterias anaerobias puede llevar a la crepitación del tejido subcutáneo (8).

\section{PRESENTACIÓN CLÍNICA}

La GF es un cuadro que se caracteriza porque en la mayor parte de los casos 
inicia con dolor perianal o perineal, que a menudo es desproporcionado al hallazgo físico, acompañado de inflamación, eritema, edema 0 prurito en el área afectada (9).

Dicha inflamación puede iniciar con la aparición de una mancha negra, llamada signo de Brodie. A partir de este momento, la inflamación necrótica se propaga enérgicamente, se mueve a lo largo de los planos fasciales y se extiende hacia las áreas circundantes (perineo, escroto, hipogastrio y, en ocasiones, afecta la región desde el muslo hasta el diafragma), lo que causa un rápido deterioro de la condición general del paciente (10).

Puede haber además datos de afectación sistémica como taquicardia, hipotensión, astenia, palidez, así como olor fétido y secreción purulenta en el área. Entre las 24 a 48 horas puede aparecer crepitación, la cual sugiere la presencia de bacterias productoras de gas como los Clostridium; sin embargo, la ausencia de esta no la excluye; conforme la infección avanza hay mionecrosis, celulitis y fascitis (7).

De acuerdo con el informe de Ersay et al, los casos de GF se presentan principalmente con dolor perianal 0 escrotal seguido de taquicardia, secreción purulenta del perineo, crepitación y fiebre. Por otro lado, Ferreira et al, informaron que la inflamación escrotal, la fiebre y el dolor eran los síntomas más comunes (3).

Las manifestaciones cutáneas macroscópicas aparecen posterior al daño iniciado a nivel del tejido subcutáneo y fascial. Por tanto, las lesiones evidentes de la piel son de menor extensión que el daño en los tejidos profundos (11).
También se pueden presentar complicaciones cardiovasculares, respiratorias, renales, metabólicas, hemorrágicas e incluso psicológicas en el postoperatorio de pacientes complicados, esto por la posible deformidad local y repercusión en la actividad sexual (12).

\section{PRONÓSTICO}

Es probable el desarrollo de sepsis y shock séptico, que junto con la falla multiorgánica son la causa más frecuente de muerte de los pacientes (13).

Existen diversos sistemas de puntuación para clasificar la severidad de la GF. Laor y sus colaboradores desarrollaron un sistema de estadificación de severidad (Índice de severidad de la Gangrena de Fournier) (ver TABLA 1), utilizando los signos vitales y datos de laboratorio. Este índice es útil en para determinar el pronóstico y la mortalidad que presentan los pacientes con GF (13). Según este estadiaje con puntuación mayor a 9 hay un $75 \%$ de mortalidad, mientras que con un puntaje menor a 9 las tasas de sobrevida son del $78 \%$ (13). Existe controversia sobre este índice, autores como Tuncel et al. no recomiendan el uso de este índice como predictor de severidad de la enfermedad, ya que no encontraron diferencias estadísticamente significativas entre su valor y la mortalidad (4).

$\mathrm{Si}$ bien la literatura reporta diversos factores con relevancia estadística para determinar la mortalidad de pacientes con GF, el índice de Laor sigue siendo la herramienta de mayor significancia a la hora de determinar la mortalidad en estos pacientes. Otro factor importante al determinar la mortalidad es la superficie corporal afectada. En contraparte es 


\begin{tabular}{|c|c|c|c|c|c|c|c|c|c|}
\hline \multirow{2}{*}{$\begin{array}{l}\text { Variables } \\
\text { Fisiológicas }\end{array}$} & \multicolumn{4}{|c|}{ Valores Anormales Elevados } & \multirow{2}{*}{$\begin{array}{c}\begin{array}{c}\text { Valores } \\
\text { Normales }\end{array} \\
0\end{array}$} & \multicolumn{4}{|c|}{ Valores Anormales Bajos } \\
\hline & +4 & +3 & +2 & +1 & & +1 & +2 & +3 & +4 \\
\hline $\begin{array}{l}\text { Temperatura } \\
{ }^{\circ} \mathrm{C}\end{array}$ & $>41$ & $39-40.9$ & - & $38.5-39.8$ & $36-37$ & $34-35.9$ & $32-33.9$ & $30-31.9$ & $<29.9$ \\
\hline $\begin{array}{c}\text { Frecuencia } \\
\text { Cardíaca (Ipm) }\end{array}$ & $>180$ & $140-179$ & $110-139$ & - & $70-109$ & - & $55-69$ & $40-54$ & $<39$ \\
\hline $\begin{array}{l}\text { Frecuencia } \\
\text { Respiratoria } \\
\text { (rpm) }\end{array}$ & $>50$ & $35-49$ & - & $25-34$ & $12-24$ & $10-11$ & $6-9$ & - & $<5$ \\
\hline $\begin{array}{l}\text { Sodio Sérico } \\
(\mathrm{mmol} / \mathrm{L})\end{array}$ & $>180$ & $160-179$ & $155-159$ & $150-154$ & $130-149$ & - & $120-129$ & $111-119$ & $<110$ \\
\hline Potasio Sérico & $>7$ & $6-6.9$ & - & $5.5-5.9$ & $3.5-5.4$ & 3-3.4 & $2.5-2.9$ & - & $<2.5$ \\
\hline $\begin{array}{l}\text { Creatinina } \\
\text { Sérica } \\
(\mathrm{mg} / 100 \mathrm{~mL}, \mathrm{si} \\
\text { falla renal } \\
\text { aguda } \times 2)\end{array}$ & $>3.5$ & $2-3.4$ & $1.5-1.9$ & - & $0.6-1.4$ & - & $<0.6$ & - & - \\
\hline $\begin{array}{l}\text { Hematocrito } \\
(\%)\end{array}$ & $>60$ & - & $50-59.9$ & $46-49.9$ & $30-45.9$ & - & $20-29.9$ & - & $<20$ \\
\hline $\begin{array}{l}\text { Leucocitos } \\
\text { (total/mm3x } \\
1000)\end{array}$ & $>40$ & - & 20-39.9 & $15-19.9$ & $3-14.9$ & - & $1-2.9$ & - & $<1$ \\
\hline $\begin{array}{c}\text { Bicarbonato } \\
\text { (venoso, } \\
\mathrm{mmol} / \mathrm{L} \text { ) }\end{array}$ & $>52$ & $41-51.9$ & - & $32-40.9$ & $22-31.9$ & - & $18-21.9$ & $15-17.9$ & $<15$ \\
\hline
\end{tabular}

importante recalcar que, pese a que la diabetes mellitus es la enfermedad de base que con mayor frecuencia se reporta, esta no tiene significancia estadística para determinar la mortalidad de los pacientes (4).

Por otra parte, Tsung-Yen et al. tras descubrir que la mayoría de los estudios mostraron que solo algunos de estos factores se asociaron con mayor mortalidad, hicieron un estudio en el 2014, en el que aplican el "Índice de Severidad de la Gangrena de Fournier Simplificado", este toma como parámetros los niveles séricos de creatinina, potasio y hematocrito, los cuales parece que están significativamente asociados con la mortalidad del paciente, además son rápidos y fáciles de usar en el diagnóstico inicial. Agregado a esto, en este estudio concluyeron que la enfermedad renal en pacientes con GF es determinante en los índices de severidad y mortalidad (14).

\section{DIAGNÓSTICO}

La sospecha de la GF es clínica, pero el diagnóstico certero, es histológico, al 
comprobar la presencia de fascitis necrotizante (13).

Dicha sospecha clínica de GF se basa principalmente en los hallazgos clínicos de fluctuación, crepitación, sensibilidad localizada y heridas de los genitales y el perineo. En la mayoría de los casos, los estudios imaginológicos no son necesarios ni deseables; bajo ninguna circunstancia, la cirugía debe retrasarse significativamente para obtener imágenes de cualquier tipo. Sin embargo, las modalidades de imagen pueden ser útiles en casos en que la presentación es atípica 0 cuando existe duda con respecto a la verdadera extensión de la enfermedad (15).

Dentro de los métodos de imagen que se utilizan en el diagnóstico de GF están la radiografía, la ecografía, la tomografía computarizada y la resonancia magnética (16).

La radiografía puede detectar enfisema subcutáneo, sin embargo la ausencia de este, que se demuestra en el $10 \%$ de los pacientes, no excluye el diagnóstico de GF $(15,16)$.

Los hallazgos del ultrasonido que sugieren GF incluyen focos hiperecoicos que muestran artefactos, lo que causa un sombreado "sucio", que representa gas en los tejidos; además el ultrasonido es útil para diferenciar GF de hernias inguinales (15).

La tomografía computarizada, juega un papel importante en el diagnóstico, dentro de los hallazgos de la tomografía computarizada se incluyen engrosamiento fascial asimétrico, colecciones de líquido, formación de abscesos, formación de cadenas de grasa alrededor de las estructuras involucradas y enfisema subcutáneo, aparte de esto es útil en la evaluación de la extensión de la enfermedad, para guiar el tratamiento quirúrgico adecuado (15).

La resonancia magnética ofrece un importante complemento diagnóstico en el manejo de la GF, esta es más útil que la radiografía convencional para especificar el rango de infección e incluso es más útil que la tomografía computarizada para planificar cualquier intervención quirúrgica (15).

\section{TRATAMIENTO}

El tratamiento de la GF requiere desbridamiento quirúrgico rápido y agresivo de los tejidos necróticos, esto además de tratamiento antibiótico de amplio espectro (3).

El tratamiento con antibióticos de amplio espectro (penicilina, metronidazol y cefalosporina de tercera generación más gentamicina) debe administrarse antes de la cirugía y debe corroborarse dicha cobertura según los resultados de los análisis de cultivos. Durante el desbridamiento quirúrgico, todas las áreas necróticas deben eliminarse y los desbridamientos deben repetirse si la necrosis continúa (3).

Una parte importante de la terapia de GF es la buena higiene local, los vendajes de las heridas deben cambiarse mínimo dos veces al día, esto además de la administración de analgésicos y antipiréticos (10).

Es esencial monitorizar de cerca a los pacientes para detectar signos tempranos de Síndrome de Dificultad Respiratoria Aguda e Insuficiencia Renal, así como para la detección y tratamiento temprano de otras posibles complicaciones. Además, en casos severos, debido a la extensa pérdida de tejido y la sepsis concomitante, algunos 
pacientes tienen necesidades metabólicas incrementadas, que se tratan mejor con nutrición parenteral (10). No es infrecuente que se requiera de orquiectomía, cistostomía o colostomía de desviación, dependiendo de la extensión de la infección, si esta ha llegado al escroto, el área perineal o la pared abdominal inferior, respectivamente (8).

En caso de shock séptico, es indispensable la reposición con abundantes líquidos, cristaloides y coloides de ser necesario, además la infusión con vasopresores, sin dejar de lado la cobertura con antimicrobianos de amplio espectro (17).

La literatura expone que lo primordial en el manejo de la GF es la cirugía, sin embargo, en pacientes que tienen contraindicación para procedimientos quirúrgicos, existen terapias menos invasivas tales como la terapia con oxígeno hiperbárico y medicamentos especiales que aceleren el crecimiento tisular, los cuales pueden ser útiles, especialmente en pacientes con un Índice de Laor $<9$.

\section{CONCLUSIÓN}

La Gangrena de Fournier es un cuadro patológico poco frecuente pero potencialmente mortal, en el que la sospecha clínica tiene una predicción elevada del mismo, por lo que un buen examen físico e historia clínica detallados son fundamentales, además de tener en cuenta las comorbilidades del paciente que muchas veces influyen en el pronóstico del mismo.

Ante la sospecha de un paciente con Gangrena de Fournier lo más importante es hacer el diagnóstico temprano, esto dada la rapidez con la que evolucionan y se deterioran estos pacientes, por tanto la demora en su diagnóstico puede definir la vida o la muerte en quienes la presentan.

Con esta revisión se concluye que, ante un caso de Gangrena de Fournier, por lo aparatoso del cuadro, es vital el diagnóstico temprano que permita así iniciar inmediatamente el tratamiento adecuado para estos pacientes; dicho abordaje debe ser agresivo, sin escatimar en opciones terapéuticas tanto médicas como quirúrgicas, con cobertura antibiótica de amplio espectro así como debridamiento del sitio afectado $y$, en pacientes que tengan alguna contraindicación para ser sometidos a cirugía, ofrecerles otras opciones de tratamiento tales como la terapia con oxígeno hiperbárico, esto con el fin de mejorar la expectativa de vida en estos pacientes.

\section{AGRADECIMIENTO}

Se agradece a la Dra. Mariana Solís por la contribución a esta revisión.

\section{REFERENCIAS}

1. Althunayyan S, Karamitosos E. Fournier's Gangrene in an Obese Female in Third Trimester of Pregnancy. Saudi Medical Journal. 2018; 39(4): 415-418.https://doi.org/10.15537/smj.2018.4.21780 
2. Romero Cabello R, Gracida Mancilla N, Romero Feregrino R, Romero Feregrino R. A case report of a woman with Fournier's gangrene and morbid obesity. Revista Latinoamericana de Patología Clínica. 2016; 63(2): 82-86. Disponible en: https://www.medigraphic.com/pdfs/patol/pt-2016/pt162e.pdf

3. Oguz A, Gümüş $M$, Turkoglu A, Bozdağ Z, Veli Ülger B, Agaçayak E et al. Fournier's Gangrene: A Summary of 10 Years of Clinical Experience. Official Journal of the Internacional College of Surgeons. 2015; 100(5): 934-941. https://doi.org/10.9738/INTSURG-D-15-00036.1

4. Camargo L, García Perdomo H. Gangrena de Fournier: revisión de factores determinantes de mortalidad. Revista Chilena de Cirugía. 2016; 68(3): 273-277.https://doi.org/10.1016/.j.rchic.2015.11.003

5. Yildiz H, Yombi J. Necrotizing Soft-Tissue Infections. New England Journal of Medicine. 2018; 378(10): 970-971. https://doi.org/10.1056/NEJMc1800049

6. Horn C, Wesp B, Fiore N, Rasane R, Torres M, Turnbull I et al. Fungal Infections Increase the Mortality Rate Three-Fold in Necrotizing Soft-Tissue Infections. Surgical Infection Society Article. 2017; 18(7): 793798. https://doi.org/10.1089/sur.2017.164

7. Devia M, García Casilimas G, Rodríguez Vera A, Jaramillo M, Redondo Á, Flórez P et al. Gangrena de fournier en una mujer joven, reporte de caso y revisión de la literatura. Revista Médica Sanitas. 2016; 19(3): 151-158. Disponible en:http://www.unisanitas.edu.co/Revista/60/GANGRENA DE FOURNIER EN UNA MUJER JOVEN R EPORTE DE CASO Y REVISION DE LA LITERATURA.pdf

8. Misiakos E, Bagias G, Patapis P, Sotiropoulos D, Kanavidis P, Machairas A. Current Concepts in the Management of Necrotizing Fasciitis. Frontiers in Surgery. 2014; 1: 00036.https://doi.org/10.3389/fsurg.2014.00036

9. Zagli G, Cianchi G, Degl'Innocenti S, Parodo J, Bonetti L, Prosperi P et al. Treatment of Fournier's Gangrene with Combination of Vacuum-Assisted Closure Therapy, Hyperbaric Oxygen Therapy, and Protective Colostomy. Case Report in Anestesiology. 2011; 2011: Article ID 430983. https://doi.org/10.1155/2011/430983

10. Kuzaka B, Wróblewska M, Borkowski T, Kawecki D, Kuzaka P, Młynarczyk G et al. Fournier’s Gangrene: Clinical Presentation of 13 Cases. Medical Science Monitor. 2018; 24: 548555. https://doi.org/10.12659/MSM.905836

11. Zárate A, Ortiz D. Gangrena de Fournier [internet]. Providencia: Facultad de Medicina UFT; 2014 [citado 14 de febrero de 2019]. Disponible en:http://www.medfinis.cl/img/manuales/gangrena.pdf

12. Muñoz Medina C, Pérez Herrera K, Luisa C. Gangrena de Fournier: reporte de un caso. Revista Hispanoamericana de Ciencias de la Salud. 2018; (3): 103-106. Disponibler en:http://www.uhsalud.com/index.php/revhispano/article/view/312

13. Rodríguez Vera A, Larios García C, García Casilimas G, Rodríguez Sabogal I, López Pérez J. Gangrena de Fournier. Revista Médica Sanitas. 2015; 18(4): 212-219. Disponible en:http://www.unisanitas.edu.co/Revista/57/GANGRENA DE FOURNIER.pdf

14. Lin T, Ou C, Tzai T, Tong $\mathrm{Y}$, Chang $\mathrm{C}$, Cheng $\mathrm{H}$ et al. Validation and simplification of Fournier's gangrene severity index. International Journal of Urology. 2014; 21: 696-701.https://doi.org/10.1111/iju.12426

15. Chennamsetty A, Khourdaji I, Burks F, Killinger K. Contemporary diagnosis and management of Fournier's gangrene. Therapeutic Advances in Urology. 2015; 7(4): 203215.https://doi.org/10.1177/1756287215584740

16. Chernyadyev S, Ufimtseva M, Vishnevskaya I, Bochkarev $Y$, Ushakov A, Beresneva $T$ et al. Fournier's Gangrene: Literature Review and Clinical Cases. Urologia Internationalis. 2018; 101(1): 9197. https://doi.org/10.1159/000490108

17. Barquero Argüello M. Las Bases de la Gangrena de Fournier. Revista Médica de Costa Rica y $\begin{array}{lllll}\text { Centroamérica. } & 2016 ; & 73 & \text { 343-346. } & \text { Disponible }\end{array}$ en:https://www.binasss.sa.cr/revistas/rmcc/619/art25.pdf 